\title{
On approximate inverse systems and resolutions
}

\author{
by
}

\author{
S. Mardešić (Zagreb)
}

\begin{abstract}
Recently, L. R. Rubin, T. Watanabe and the author have introduced approximate inverse systems and approximate resolutions, a new tool designed to study topological spaces. These systems differ from the usual inverse systems in that the bonding maps $p_{a a^{\prime}}$ are not subject to the commutativity requirement $p_{a a^{\prime}} p_{a^{\prime} a^{\prime \prime}}=p_{a a^{\prime \prime}}, a \leq a^{\prime}$ $\leq a^{\prime \prime}$. Instead, the mappings $p_{a a^{\prime}} p_{a^{\prime} a^{\prime \prime}}$ and $p_{a a^{\prime \prime}}$ are allowed to differ in a way controlled by coverings $\mathcal{U}_{a}$, called meshes, which are associated with the members $X_{a}$ of the system. The purpose of this paper is to consider a more general and much simpler notion of approximate system and approximate resolution, which does not require meshes. The main result is a construction which associates with any approximate resolution in the new sense an approximate resolution in the previous sense in such a way that previously obtained results remain valid in the present more general setting.
\end{abstract}

1. Introduction. The technique of inverse limits is widely used in the topology of compact spaces, but it is of little use in non-compact situations. Having this in mind, in 1981 the author introduced resolutions [3] (see also [6]), which can be viewed as special cases of inverse limits and behave in the non-compact case just as inverse limits behave in the compact case. A resolution of a space $X$ consists of an inverse system of spaces and mappings $\boldsymbol{X}=\left(X_{a}, p_{a a^{\prime}}, A\right)$ and of a system of mappings $\boldsymbol{p}: X \rightarrow \boldsymbol{X}$ which satisfy conditions (R1) and (R2) stated below. In 1989 L. R. Rubin and the author [5] introduced approximate inverse systems of compacta and their limits by relaxing the usual commutativity requirement on the bonding mappings. Both ideas were put together by T. Watanabe and the author in [7] to give the notion of an approximate resolution of a space $X$. It consists of an approximate inverse system $\mathcal{X}$ and of a system of mappings $\boldsymbol{p}: X \rightarrow \mathcal{X}$ which satisfy conditions (R1) and (R2).

In describing these notions precisely, we use the following notation.

1991 Mathematics Subject Classification: 54B35, 54F45.

Key words and phrases: inverse system, approximate inverse system, inverse limit, resolution, approximate resolution, dimension. 
$\operatorname{Cov}(X)$ is the set of all normal coverings of a topological space $X$. If $\mathcal{U} \in \operatorname{Cov}(X)$ and $A \subseteq X$, then $\operatorname{st}(A, \mathcal{U})$ denotes the union of all $U \in \mathcal{U}$ such that $U \cap A \neq \emptyset$. We denote by $\operatorname{st}(\mathcal{U})$ the covering formed by all sets

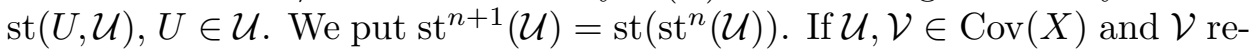
fines $\mathcal{U}$, we write $\mathcal{V} \prec \mathcal{U}$. If $\operatorname{st}(\mathcal{V})$ refines $\mathcal{U}$ we say that $\mathcal{V}$ is a star-refinement of $\mathcal{U}$. If $f, f^{\prime}: Y \rightarrow X$ are $\mathcal{U}$-near mappings, we write $\left(f, f^{\prime}\right) \prec \mathcal{U}$.

An approximate inverse system was defined in [7] (see also [5]) as a collection $\mathcal{X}=\left(X_{a}, \mathcal{U}_{a}, p_{a a^{\prime}}, A\right)$, where $(A, \leq)$ is an unbounded directed set, $X_{a}, a \in A$, is a topological space, $\mathcal{U}_{a}, a \in A$, is a normal covering of $X_{a}$, called the mesh of $X_{a}$ and $p_{a a^{\prime}}: X_{a^{\prime}} \rightarrow X_{a}, a \leq a^{\prime}$, are mappings, called the bonding mappings. Three conditions were imposed:

(A1) $\left(p_{a a_{1}} p_{a_{1} a_{2}}, p_{a a_{2}}\right) \prec \mathcal{U}_{a}, a \leq a_{1} \leq a_{2}, p_{a a}=$ id.

(A2) For any $a \in A$ and any $\mathcal{U} \in \operatorname{Cov}\left(X_{a}\right)$, there exists an $a^{\prime} \geq a$ such that $\left(p_{a a_{1}} p_{a_{1} a_{2}}, p_{a a_{2}}\right) \prec \mathcal{U}$, for $a_{2} \geq a_{1} \geq a^{\prime}$.

(A3) For any $a \in A$ and any $\mathcal{U} \in \operatorname{Cov}\left(X_{a}\right)$, there exists an $a^{\prime} \geq a$ such that $\mathcal{U}_{a^{\prime \prime}} \prec\left(p_{a a^{\prime \prime}}\right)^{-1}(\mathcal{U})$, for each $a^{\prime \prime} \geq a^{\prime}$.

In this paper we refer to approximate systems defined as above as to gauged approximate systems. The main purpose of the paper is to consider the following more general and much simpler notion of an approximate system, which does not use meshes and which was introduced by M. G. Charalambous for systems of uniform spaces [1].

Definition 1. An approximate inverse system is a collection $\boldsymbol{X}=$ $\left(X_{a}, p_{a a^{\prime}}, A\right)$, where $(A, \leq)$ is a directed preordered set, $X_{a}, a \in A$, is a topological space and $p_{a a^{\prime}}: X_{a^{\prime}} \rightarrow X_{a}, a \leq a^{\prime}$, are mappings such that $p_{a a}=\mathrm{id}$ and condition (A2) is satisfied.

Other basic notions, defined in [7], include approximate mappings of a space into an approximate system, the limit of an approximate system and approximate resolutions of a space. These notions referred to gauged approximate systems, i.e. systems endowed with meshes, but made no explicit use of meshes. Therefore, in the present paper we adopt these definitions with no changes.

Definition 2. An approximate mapping $\boldsymbol{f}: Z \rightarrow \boldsymbol{X}$ of a space $Z$ into an approximate system is a collection of mappings $f_{a}: Z \rightarrow X_{a}, a \in A$, such that the following condition holds:

(AS) For any $a \in A$ and any $\mathcal{U} \in \operatorname{Cov}\left(X_{a}\right)$, there exists an $a^{\prime} \geq a$ such that $\left(p_{a a^{\prime \prime}} f_{a^{\prime \prime}}, f_{a}\right) \prec \mathcal{U}$, for each $a^{\prime \prime} \geq a^{\prime}$.

Definition 3. A limit of an approximate system $\boldsymbol{X}$ is an approximate mapping $\boldsymbol{p}: X \rightarrow \boldsymbol{X}$ of a space $X$ which has the following universal property: 
(UL) For any approximate mapping $\boldsymbol{f}=\left(f_{a}, a \in A\right): Z \rightarrow \boldsymbol{X}$ of a space $Z$, there exists a unique mapping $\phi: Z \rightarrow X$ such that $p_{a} \phi=f_{a}$, for each $a \in A$.

Note that this definition immediately implies uniqueness of limits (up to natural homeomorphism).

Definition 4. An approximate resolution of a space $X$ consists of an approximate system $\boldsymbol{X}$ and of an approximate mapping $\boldsymbol{p}=\left(p_{a}\right): X \rightarrow \boldsymbol{X}$ with the following properties:

(R1) For any polyhedron $P$, open covering $\mathcal{V}$ of $P$ and mapping $f: X \rightarrow$ $P$, there exists an $a \in A$ such that for any $a^{\prime} \geq a$ there exists a mapping $g: X_{a^{\prime}} \rightarrow P$ satisfying $\left(g p_{a^{\prime}}, f\right) \prec \mathcal{V}$.

$(\mathrm{R} 2)$ For any polyhedron $P$ and open covering $\mathcal{V}$ of $P$, there exists a $\mathcal{V}^{\prime} \in \operatorname{Cov}(P)$ such that, for any $a \in A$ and mappings $g, g^{\prime}: X_{a} \rightarrow P$ which satisfy $\left(g p_{a}, g^{\prime} p_{a}\right) \prec \mathcal{V}^{\prime}$, there exists an $a^{\prime} \geq a$ such that $\left(g p_{a a^{\prime \prime}}, g^{\prime} p_{a a^{\prime \prime}}\right) \prec \mathcal{V}$, for each $a^{\prime \prime} \geq a$.

In this paper we use boldface characters to denote approximate systems and approximate resolutions in the sense of the above definitions, while gauged approximate systems and gauged approximate resolutions are denoted by script characters.

R e mark 1. Obviously, every usual inverse system $\boldsymbol{X}$ is an approximate inverse system in the sense of Definition 1 and every resolution $\boldsymbol{p}: X \rightarrow \boldsymbol{X}$ is an approximate resolution in the sense of Definition 4.

Our main results show that theorems proved before for gauged approximate systems and gauged approximate resolutions also hold for approximate systems and approximate resolutions in the sense of this paper. These results are derived by using a construction which assigns to any approximate system $\boldsymbol{X}$ a closely related gauged approximate system $\mathcal{X}$, called the induced gauged approximate system. A statement proved valid for gauged systems is valid for $\mathcal{X}$, which implies its validity for $\boldsymbol{X}$.

Following [5], M. G. Charalambous [1] recently developed a theory of approximate inverse systems of uniform spaces using only property (A2). This was one of the reasons why the author attempted to clarify the relationship between approximate systems satisfying only condition (A2) and gauged systems, i.e. systems satisfying conditions (A1)-(A3). Some of Charalambous' results are closely related to some of ours (e.g., to our Theorem 11).

In [7], beside gauged approximate systems $\mathcal{X}$ and resolutions $\boldsymbol{p}: X \rightarrow \mathcal{X}$ also approximate mappings $f=\left(f, f_{b}\right): \mathcal{X} \rightarrow \mathcal{Y}$ between such systems were defined. Then an equivalence relation between approximate mappings $\boldsymbol{f}=\left(f, f_{b}\right): \mathcal{X} \rightarrow \mathcal{Y}$ was introduced, thus yielding equivalence classes of 
approximate mappings $[\boldsymbol{f}]$. Moreover, for cofinite resolutions consisting of topologically complete spaces a composition of equivalence classes of approximate mappings was defined. It was proved that in this way one obtains a category denoted by APRES [7, Theorem 8.12]. Finally, it was shown that the restriction APRES POL $_{P O}$ of this category to systems which consist of polyhedra is a category equivalent to the category of topologically complete spaces and continuous mappings [7, Theorem 8.13].

It is natural to ask whether analogous results also hold for approximate systems in the sense of the present paper, i.e. for systems satisfying only condition (A2). These questions are studied in detail in a comprehensive paper by V. Matijević and N. Uglešić [8]. They define approximate mappings $\boldsymbol{f}=\left(f, f_{b}\right): \boldsymbol{X} \rightarrow \boldsymbol{Y}$ and their equivalence classes in a natural way. However, when they tried to define composition of morphisms and prove the key results for systems of polyhedra, they were forced to introduce an additional condition on the systems, which itself implies that the systems can be endowed with meshes in such a manner that they become systems in the sense of [7]. After all, in [9] Watanabe introduced meshes in order to be able to organize usual (commutative) resolutions and their approximate mappings into a category. On the other hand, there are many problems in topology where there is no need for mappings. This justifies studying non-gauged systems even if one does not have a category.

2. The main construction. In this section we describe the main construction and establish its basic properties, listed in the following theorem.

Theorem 1. Let $\boldsymbol{X}=\left(X_{a}, p_{a a^{\prime}}, A\right)$ be an approximate system. Then there exist a gauged approximate system $\mathcal{X}=\left(Y_{b}, \mathcal{U}_{b}, q_{b b^{\prime}}, B\right)$ and an increasing function $s: B \rightarrow A$ with the following properties:

(i) $B$ is antisymmetric, directed, unbounded and cofinite.

(ii) $Y_{b}=X_{s(b)}, q_{b b^{\prime}}=p_{s(b) s\left(b^{\prime}\right)}, b, b^{\prime} \in B, b \leq b^{\prime}$.

(iii) $s: B \rightarrow A$ is surjective.

For any such gauged system $\mathcal{X}$ we say that it is induced by $\boldsymbol{X}$.

Proof. In the proof we use some ideas from a recent paper by Vlasta Matijević and the author [4]. The index set $B$ is defined as the union of a sequence of infinite, ordered, antisymmetric, cofinite sets $\emptyset \subseteq B_{0} \subseteq B_{1} \subseteq \ldots$. such that the restriction of the ordering of $B_{n}$ to $B_{n-1}$ coincides with the ordering of $B_{n-1}$. The ordering of $B$ is the unique ordering whose restriction to each $B_{n}$ yields the ordering of $B_{n}$. In order to define $s: B \rightarrow A$, we will define a sequence of increasing functions $s_{n}: B_{n} \rightarrow A$ such that $s_{n}$ extends $s_{n-1}$. Then $s$ is the only function with $s \mid B_{n}=s_{n}$, for all $n$. Clearly, $s$ defined in this way is an increasing function. Once $s(b), s\left(b^{\prime}\right)$ are defined, 
we define the spaces $Y_{b}$ and the mappings $q_{b b^{\prime}}$ by (ii). We must also define an open covering $\mathcal{U}_{b} \in \operatorname{Cov}\left(Y_{b}\right)$, for each $b \in B$.

The ordered set $B$ has a special structure which we now describe. The set $C_{n}=B_{n} \backslash B_{n-1}$ is the set of all maximal elements of $B_{n}$; it is an infinite set, for $n \geq 0$. If $i<j, c_{i} \in C_{i}, c_{j} \in C_{j}$ and $c_{i}<c_{j}$, i.e. $c_{i} \leq c_{j}$ and $c_{i} \neq c_{j}$, we require the existence of a chain $c_{i}<c_{i+1}<\ldots<c_{j}$, where $c_{k} \in C_{k}$, $i \leq k \leq j$. Finally, we require that for any two elements $c_{n-1}, c_{n-1}^{\prime} \in C_{n-1}$, there exists an element $c_{n} \in C_{n}$ such that $c_{n-1}<c_{n}$ and $c_{n-1}^{\prime}<c_{n}$. Clearly, $B$ constructed in this way is a cofinite unbounded directed ordered set.

We will also define, for each pair $c_{i} \in C_{i}, c_{i+1} \in C_{i+1}, c_{i}<c_{i+1}$, a covering $\mathcal{V}_{c_{i} c_{i+1}} \in \operatorname{Cov}\left(Y_{c_{i}}\right)$. The sets $B_{n}$, the functions $s_{n}=s \mid B_{n}$, the coverings $\mathcal{U}_{b}, b \in B_{n}$, and the coverings $\mathcal{V}_{c_{i} c_{i+1}}, i+1 \leq n$, will be defined by induction on $n$. We require that these objects satisfy the following conditions:

(1) $\quad\left(p_{s(b) s\left(b_{1}\right)} p_{s\left(b_{1}\right) a^{\prime}}, p_{s(b) a^{\prime}}\right) \prec \mathcal{U}_{b}$,

$$
b, b_{1} \in B_{n}, a^{\prime} \in A, b \leq b_{1}, s\left(b_{1}\right) \leq a^{\prime},
$$

$$
\begin{aligned}
&\left(p_{s\left(c_{i}\right) a^{\prime}} p_{a^{\prime} a^{\prime \prime}}, p_{s\left(c_{i}\right) a^{\prime \prime}}\right) \prec \mathcal{V}_{c_{i} c_{i+1}}, \\
& i+1 \leq n, a^{\prime}, a^{\prime \prime} \in A, s\left(c_{i+1}\right) \leq a^{\prime} \leq a^{\prime \prime}, \\
& \mathcal{U}_{b^{\prime \prime}} \prec\left(p_{s\left(c_{i}\right) s\left(b^{\prime \prime}\right)}\right)^{-1}\left(\mathcal{V}_{c_{i} c_{i+1}}\right), \quad b^{\prime \prime} \in B_{n}, c_{i+1} \leq b^{\prime \prime} .
\end{aligned}
$$

Finally, we require that for any $c_{i} \in C_{i}$, and any covering $\mathcal{V}_{c_{i}} \in \operatorname{Cov}\left(Y_{c_{i}}\right)$, there exists a $c_{i+1} \in C_{i+1}$ such that

$$
c_{i}<c_{i+1}, \quad \mathcal{V}_{c_{i} c_{i+1}}=\mathcal{V}_{c_{i}}, \quad i+1 \leq n .
$$

We begin the induction by choosing for $B_{0}=C_{0}$ any infinite set and for $s_{0}: B_{0} \rightarrow A$ a surjective function. We consider $B_{0}$ to be totally unordered. For $b \in B_{0}$, we take for $\mathcal{U}_{b}$ any normal covering of $Y_{b}$. Let us now assume that we have already defined all the data referring to integers $\leq n$. In the induction step to $n+1$, we first define $C_{n+1}$ as the set of all ordered quadruples

$$
c_{n+1}=\left(c_{n}, c_{n}^{\prime}, \mathcal{V}_{c_{n}}, \mathcal{V}_{c_{n}^{\prime}}\right),
$$

where $c_{n}, c_{n}^{\prime}$ are different elements of $C_{n}$ and $\mathcal{V}_{c_{n}}, \mathcal{V}_{c_{n}^{\prime}}$ are arbitrary open coverings of $Y_{c_{n}}$ and $Y_{c_{n}^{\prime}}$ respectively. We then put

$$
B_{n+1}=B_{n} \cup C_{n+1} .
$$

The ordering on $B_{n+1}$ is defined so that it agrees with the ordering already defined on $B_{n}$. The set $C_{n+1} \subseteq B_{n+1}$ is considered to be totally unordered. For $c_{n+1}$ as in (5), we put $c_{n}<c_{n+1}, c_{n}^{\prime}<c_{n+1}$ and consider $c_{n}$ and $c_{n}^{\prime}$ to be the only predecessors of $c_{n+1}$ belonging to $C_{n}$. For $b \in B_{n-1}$, we put $b<c_{n+1}$ if and only if $b<c_{n}$ or $b<c_{n}^{\prime}$. Clearly, this defines an ordering on $B_{n+1}$ which has all the desired properties. 
For $c_{n+1}$ as in (5), we put

$$
\mathcal{V}_{c_{n} c_{n+1}}=\mathcal{V}_{c_{n}}, \quad \mathcal{V}_{c_{n}^{\prime} c_{n+1}}=\mathcal{V}_{c_{n}^{\prime}}
$$

Since $C_{n}$ is infinite, for a given $c_{n} \in C_{n}$, we can choose a $c_{n}^{\prime} \in C_{n}$ which differs from $c_{n}$. Then (7) yields a $c_{n+1}$ which satisfies (4).

Now we must extend $s \mid B_{n}: B_{n} \rightarrow A$ to $s \mid B_{n+1}: B_{n+1} \rightarrow A$ by defining $s\left(c_{n+1}\right) \in A$, for $c_{n+1} \in C_{n+1}$. First note that $c_{n+1}$ has finitely many predecessors $b \in B_{n}, b<c_{n+1}$. Therefore, by (A2) applied to $\boldsymbol{X}$, there exists an $a \in A$ such that $a \geq s(b)$, for $b<c_{n+1}$, and

$$
\left(p_{s(b) a^{\prime}} p_{a^{\prime} a^{\prime \prime}}, p_{s(b) a^{\prime \prime}}\right) \prec \mathcal{U}_{b}, \quad a^{\prime}, a^{\prime \prime} \in A, a \leq a^{\prime} \leq a^{\prime \prime} .
$$

For a given $c_{n+1} \in C_{n+1}$, there are only two elements $c_{n} \in C_{n}, c_{n}<c_{n+1}$. Therefore, using (A2) for $\boldsymbol{X}$, one can achieve that

$$
\left(p_{s\left(c_{n}\right) a^{\prime}} p_{a^{\prime} a^{\prime \prime}}, p_{s\left(c_{n}\right) a^{\prime \prime}}\right) \prec \mathcal{V}_{c_{n} c_{n+1}}, \quad a^{\prime}, a^{\prime \prime} \in A, a \leq a^{\prime} \leq a^{\prime \prime} .
$$

Now define $s\left(c_{n+1}\right)=a$. Formulas (8) and (9) show that (1) and (2) remain valid also for $n+1$. Since each $b^{\prime \prime} \in B_{n}$ has only finitely many predecessors, it is possible to choose a sufficiently fine covering $\mathcal{U}_{b^{\prime \prime}}$ of $Y_{b^{\prime \prime}}$ so that (3) remains valid also for $n+1$.

It is now easy to verify that $\mathcal{X}$ is a gauged approximate system. Indeed, condition (A1) follows from (1) by putting $a^{\prime}=s\left(b_{2}\right)$, because $b \leq b_{1} \leq b_{2}$ implies $s\left(b_{1}\right) \leq s\left(b_{2}\right)=a^{\prime}, p_{s(b) s\left(b_{1}\right)}=q_{b b_{1}}, p_{s\left(b_{1}\right) a^{\prime}}=q_{b_{1} b_{2}}, p_{s(b) a^{\prime}}=q_{b b_{2}}$. In order to verify condition (A2), consider any $b=c_{i} \in C_{i} \subseteq B$ and any covering $\mathcal{U} \in \operatorname{Cov}\left(Y_{c_{i}}\right)$. By (4), there exists a $c_{i+1} \in C_{i+1}$ such that $c_{i}<$ $c_{i+1}$ and $\mathcal{V}_{c_{i} c_{i+1}}=\mathcal{U}$. Then (2) shows that (A2) holds for $b^{\prime}=c_{i+1}$. Indeed, $b<b^{\prime}$ and $b^{\prime} \leq b_{1} \leq b_{2}$ imply $s\left(b^{\prime}\right) \leq a^{\prime}=s\left(b_{1}\right) \leq a^{\prime \prime}=s\left(b_{2}\right)$. Moreover, $p_{s\left(c_{i}\right) a^{\prime}}=q_{b b_{1}}, p_{a^{\prime} a^{\prime \prime}}=q_{b_{1} b_{2}}, p_{s\left(c_{i}\right) a^{\prime \prime}}=q_{b b_{2}}$. In order to verify condition (A3), consider $b \in B$ and any covering $\mathcal{U} \in \operatorname{Cov}\left(Y_{b}\right)$. As above, choose $c_{i+1} \in C_{i+1}$ so that $b=c_{i}<c_{i+1}$ and $\mathcal{V}_{c_{i} c_{i+1}}=\mathcal{U}$. Then, for $b^{\prime}=c_{i+1}$ and $b^{\prime \prime} \in B, b^{\prime \prime} \geq b^{\prime},(3)$ applies and yields (A3), as desired. Condition (iii) is satisfied, because already $s \mid B_{0}=s_{0}$ is a surjection onto $A$.

Remark 2. In Theorem 1 assertion (iii) can be strengthened to

(iii)' For any $a \in A$ and $\mathcal{W} \in \operatorname{Cov}\left(X_{a}\right)$, there exists an element $b \in B$ such that $a=s(b)$ and $\mathcal{W}=\mathcal{U}_{b}$.

In order to obtain also condition (iii) ${ }^{\prime}$, it suffices to change the initial step in the above construction, i.e. to modify the definition of $B_{0}, s_{0}$ and $\mathcal{U}_{b}$, for $b \in B_{0}$. We take an infinite set $D$ and a surjection $t: D \rightarrow A$. Then $B_{0}$ is the set of all pairs $b=(d, \mathcal{W})$, where $d \in D$ and $\mathcal{W} \in \operatorname{Cov}\left(X_{t(d)}\right)$. As before, we consider $B_{0}$ as totally unordered and we define $s_{0}$ by putting $s_{0}(d, \mathcal{W})=t(d)$. Finally, for $b=(d, \mathcal{W})$, we put $\mathcal{U}_{b}=\mathcal{W}$. For any $a \in A$ and $\mathcal{W} \in \operatorname{Cov}\left(X_{a}\right)$, there exists a $d \in D$ such that $t(d)=a$. Therefore, for $b=(d, \mathcal{W})$, we have $s_{0}(b)=a$ and $\mathcal{U}_{b}=\mathcal{W}$, as required by (iii) . 
3. Approximate mappings and limits. In this section we show how an approximate mapping $\boldsymbol{f}: Z \rightarrow \boldsymbol{X}$ induces an approximate mapping $\boldsymbol{g}$ : $Z \rightarrow \mathcal{X}$ and vice versa. It follows that an approximate mapping $\boldsymbol{p}: X \rightarrow \boldsymbol{X}$ is a limit if and only if the induced approximate mapping $\boldsymbol{q}: X \rightarrow \mathcal{X}$ is a limit.

THEOREM 2. Let $\boldsymbol{X}$ be an approximate system and $\mathcal{X}$ an induced gauged system (see Theorem 1). Let $\boldsymbol{f}=\left(f_{a}\right): Z \rightarrow \boldsymbol{X}$ be an approximate mapping. Then the mappings $g_{b}=f_{s(b)}: Z \rightarrow Y_{b}, b \in B$, form an approximate mapping $\boldsymbol{g}=\left(g_{b}\right): Z \rightarrow \mathcal{X}$, said to be induced by $\boldsymbol{f}$.

Proof. We must show that $\boldsymbol{g}$ satisfies condition (AS). Consider any $b \in B$ and $\mathcal{U} \in \operatorname{Cov}\left(Y_{b}\right)$. Put $a=s(b)$ and note that $Y_{b}=X_{s(b)}$. Since $\boldsymbol{f}$ has property (AS), there exists an $a^{\prime} \geq a$ such that $\left(f_{a}, p_{a a^{\prime \prime}} f_{a^{\prime \prime}}\right) \prec \mathcal{U}$, for each $a^{\prime \prime} \geq a^{\prime}$. By Theorem 1(iii), there exists a $b^{*} \in B$ such that $s\left(b^{*}\right)=a^{\prime}$. By directedness of $B$, there exists a $b^{\prime} \geq b, b^{*}$. Therefore, $b^{\prime \prime} \geq b^{\prime}$ implies $a^{\prime \prime}=s\left(b^{\prime \prime}\right) \geq s\left(b^{\prime}\right) \geq s\left(b^{*}\right)=a^{\prime}$, and we obtain the desired conclusion $\left(g_{b}, q_{b b^{\prime \prime}} g_{b^{\prime \prime}}\right)=\left(f_{s(b)}, p_{s(b) s\left(b^{\prime \prime}\right)} f_{s\left(b^{\prime \prime}\right)}\right) \prec \mathcal{U}$, for each $b^{\prime \prime} \geq b^{\prime}$.

TheOREM 3. Let $\boldsymbol{X}$ be an approximate system and $\mathcal{X}$ an induced gauged approximate system (see Theorem 1). Let $\boldsymbol{g}=\left(g_{b}\right): Z \rightarrow \mathcal{X}$ be an approximate mapping and let $t: A \rightarrow B$ be a function such that st $=\mathrm{id}$. Then the mappings $f_{a}=g_{t(a)}: Z \rightarrow X_{a}, a \in A$, form an approximate mapping $\boldsymbol{f}=\left(f_{a}\right): Z \rightarrow \boldsymbol{X}$, said to be induced by $\boldsymbol{g}$.

Proof. Note that $t$ is not assumed to be increasing, which makes the proof more difficult. We must verify condition (AS) for the collection of mappings $\left(f_{a}\right)$. Consider any $a \in A$ and $\mathcal{U} \in \operatorname{Cov}\left(X_{a}\right)$. Choose a starrefinement $\mathcal{V}$ of $\mathcal{U}$. Applying (A2) to $\boldsymbol{p}$, we obtain an $a^{\prime} \geq a$ such that

$$
\left(p_{a a_{1}} p_{a_{1} a_{2}}, p_{a a_{2}}\right) \prec \mathcal{V}, \quad a_{2} \geq a_{1} \geq a^{\prime} .
$$

We will show that $a^{\prime}$ has the property required by $(\mathrm{AS})$ for $\left(f_{a}\right)$, i.e.

$$
\left(f_{a}, p_{a a^{\prime \prime}} f_{a^{\prime \prime}}\right) \prec \mathcal{U}, \quad \forall a^{\prime \prime} \geq a^{\prime} .
$$

Indeed, let $a^{\prime \prime} \geq a^{\prime}$. Put $b=t(a), b^{\prime \prime}=t\left(a^{\prime \prime}\right), \mathcal{V}^{\prime \prime}=\left(p_{a a^{\prime \prime}}\right)^{-1}(\mathcal{V})$. By (AS) for $\boldsymbol{g}$, there exists a $b^{*} \geq b, b^{\prime \prime}$ such that

$$
\begin{aligned}
\left(g_{b}, q_{b b^{*}} g_{b^{*}}\right) & \prec \mathcal{V}, \\
\left(g_{b^{\prime \prime}}, q_{b^{\prime \prime} b^{*}} g_{b^{*}}\right) & \prec \mathcal{V}^{\prime \prime} .
\end{aligned}
$$

Note that $s(b)=s t(a)=a$ and put $a^{*}=s\left(b^{*}\right)$. Then $q_{b b^{*}}=p_{a a^{*}}$. Moreover, $g_{b}=g_{t(a)}=f_{a}$. Therefore, (3) becomes

$$
\left(f_{a}, p_{a a^{*}} g_{b^{*}}\right) \prec \mathcal{V} \text {. }
$$

Now note that $s\left(b^{\prime \prime}\right)=s t\left(a^{\prime \prime}\right)=a^{\prime \prime}$ and therefore $q_{b^{\prime \prime} b^{*}}=p_{a^{\prime \prime} a^{*}}$. Moreover, 
$f_{a^{\prime \prime}}=g_{t\left(a^{\prime \prime}\right)}=g_{b^{\prime \prime}}$. Consequently, application of $p_{a a^{\prime \prime}}$ to (4) yields

$$
\left(p_{a a^{\prime \prime}} f_{a^{\prime \prime}}, p_{a a^{\prime \prime}} p_{a^{\prime \prime} a^{*}} g_{b^{*}}\right) \prec \mathcal{V} \text {. }
$$

Next, note that $b^{*} \geq b^{\prime \prime}$ implies $a^{*}=s\left(b^{*}\right) \geq s\left(b^{\prime \prime}\right)=a^{\prime \prime}$. Since $a^{\prime \prime} \geq a^{\prime},(1)$ yields

$$
\left(p_{a a^{\prime \prime}} p_{a^{\prime \prime} a^{*}}, p_{a a^{*}}\right) \prec \mathcal{V} .
$$

Therefore, one has also

$$
\left(p_{a a^{\prime \prime}} p_{a^{\prime \prime} a^{*}} g_{b^{*}}, p_{a a^{*}} g_{b^{*}}\right) \prec \mathcal{V} .
$$

Since $\operatorname{st}(\mathcal{V}) \prec \mathcal{U},(5),(6)$ and (8) yield the desired relation (2).

We now apply Theorems 2 and 3 to limits.

TheOrem 4. Let $\boldsymbol{X}$ be an approximate system and let $\mathcal{X}$ be an induced gauged system (see Theorem 1). Let $\boldsymbol{p}=\left(p_{a}\right): X \rightarrow \boldsymbol{X}$ be an approximate mapping and let $\boldsymbol{q}=\left(q_{b}\right): X \rightarrow \mathcal{X}$ be an induced approximate mapping (see Theorem 2). If $\boldsymbol{p}$ is a limit and all $X_{a}$ are Tikhonov spaces, then $\boldsymbol{q}$ is a limit. Conversely, if $\boldsymbol{q}$ is a limit, then also $\boldsymbol{p}$ is a limit.

P r o of. First assume that $\boldsymbol{p}$ is a limit and recall that $\boldsymbol{q}$ is given by $q_{b}=$ $p_{s(b)}$. We must show that for any approximate mapping $\boldsymbol{g}=\left(g_{b}\right): Z \rightarrow \mathcal{X}$, there is a unique mapping $\phi: Z \rightarrow X$ such that

$$
q_{b} \phi=g_{b}, \quad \forall b \in B .
$$

By Theorem 3, $\boldsymbol{g}$ induces an approximate mapping $\boldsymbol{f}=\left(f_{a}\right): Z \rightarrow \boldsymbol{X}$, where $f_{a}=g_{t(a)}$ and $t: A \rightarrow B$ is a function such that $s t=\mathrm{id}$. Now the definition of an approximate mapping yields a mapping $\phi: Z \rightarrow X$ such that

$$
p_{a} \phi=f_{a}, \quad \forall a \in A .
$$

From (10), we derive

$$
q_{b} \phi=g_{t s(b)}, \quad \forall b \in B,
$$

because $q_{b} \phi=p_{s(b)} \phi=f_{s(b)}=g_{t s(b)}$. In order to obtain the desired equality (9), it suffices to show that $g_{b}=g_{t s(b)}$, for each $b \in B$. Since $Y_{b}=X_{s(b)}$ is a Tikhonov space, it suffices to show that, for each normal covering $\mathcal{U}$ of $Y_{b}$, one has

$$
\left(g_{b}, g_{t s(b)}\right) \prec \mathcal{U}
$$

However, this is an easy consequence of (AS) for $\boldsymbol{g}$. Indeed, if $\mathcal{V}$ is a starrefinement of $\mathcal{U}$, we can find a $b^{\prime} \geq b, t s(b)$ such that

$$
\left(g_{b}, q_{b b^{\prime}} g_{b^{\prime}}\right) \prec \mathcal{V}, \quad\left(g_{t s(b)}, q_{t s(b) b^{\prime}} g_{b^{\prime}}\right) \prec \mathcal{V} .
$$

Now note that $q_{t s(b) b^{\prime}}=p_{s(b) s\left(b^{\prime}\right)}=q_{b b^{\prime}}$, because $s t s=s$. Therefore, (12) follows from (13). It remains to show uniqueness of $\phi$. Assume that we 
also have a mapping $\psi: Z \rightarrow X$ with the property $q_{b} \psi=g_{b}, b \in B$. Then $f_{a}=g_{t(a)}=q_{t(a)} \psi=p_{s t(a)} \psi=p_{a} \psi$, for all $a \in A$. Consequently, $\psi=\phi$.

To prove the converse we do not need the assumption that the spaces are Tikhonov. We assume that $\boldsymbol{q}$ is a limit and need to prove that also $\boldsymbol{p}$ is a limit. We must prove that for any approximate mapping $\boldsymbol{f}=\left(f_{a}\right): Z \rightarrow \boldsymbol{X}$, there is a unique mapping $\phi: Z \rightarrow X$ which satisfies (10). Consider the induced approximate mapping $\boldsymbol{g}: Z \rightarrow \mathcal{X}$ given by the mappings $g_{b}=f_{s(b)}$ (see Theorem 2). Since $\boldsymbol{q}$ is a limit, there exists a unique mapping $\phi: Z \rightarrow X$ such that $q_{b} \phi=g_{b}$, for all $b \in B$. Since $s: B \rightarrow A$ is onto, each $a \in A$ admits a $b \in B$ such that $a=s(b)$. Therefore, $p_{a} \phi=p_{s(b)} \phi=q_{b} \phi=$ $g_{b}=f_{s(b)}=f_{a}, a \in A$. Now assume that $\psi: Z \rightarrow X$ is another mapping satisfying $p_{a} \psi=f_{a}$, for all $a \in A$. Then for any $b \in B$ and $a=s(b)$ one has $q_{b} \psi=p_{s(b)} \psi=p_{a} \psi=f_{a}=f_{s(b)}=g_{b}$. By the uniqueness of $\phi$, we conclude that $\psi=\phi$.

Corollary 1. Every approximate system of Tikhonov spaces $\boldsymbol{X}$ has a limit $\boldsymbol{p}: X \rightarrow \boldsymbol{X}, X=\lim \boldsymbol{X}$, which is unique up to natural homeomorphism.

Corollary 2. If $\boldsymbol{X}$ is a usual inverse system of Tikhonov spaces, then limits in the usual sense and in the sense of Definition 3 coincide.

Both corollaries are immediate consequences of Theorem 4 and the corresponding results for gauged approximate systems [7, Theorem 1.14 and Remark 1.15].

TheOREM 5. The limit of an approximate system $\boldsymbol{X}$ of topologically complete spaces is topologically complete.

Proof. Applying Theorem 4 to a limit $\boldsymbol{p}: X \rightarrow \boldsymbol{X}$, we conclude that the induced approximate mapping $\boldsymbol{p}: X \rightarrow \mathcal{X}$ is also a limit. Since all the members of $\mathcal{X}$ are topologically complete spaces $Y_{b}=X_{s(a)}$ and the corresponding result holds for gauged approximate systems [7, Theorem 1.17], we conclude that $X$ is topologically complete.

Similarly, using [7, Theorem 4.1], one obtains the following result.

TheOREM 6. The limit of an approximate system $\boldsymbol{X}$ of (non-empty) compact Hausdorff spaces is a (non-empty) compact Hausdorff space.

4. Approximate resolutions. All results in this section will be derived from the following theorem.

Theorem 7. Let $\boldsymbol{p}=\left(p_{a}\right): X \rightarrow \boldsymbol{X}$ be an approximate mapping and let $\boldsymbol{q}=\left(q_{b}\right): X \rightarrow \mathcal{X}$ be an induced approximate mapping (see Theorem 2). Then $\boldsymbol{p}$ is a resolution if and only if $\boldsymbol{q}$ is a gauged approximate resolution. 
T. Watanabe proved the analogous result for (commutative) resolutions and approximate resolutions in his sense [9, Proposition 3.7]. Note that his approximate resolutions are commutative and therefore a very special case of our approximate resolutions.

We precede the proof by introducing and analyzing a formally weaker, but equivalent, form of properties (R1) and (R2) for an approximate mapping $\boldsymbol{p}: X \rightarrow \boldsymbol{X}$.

$(\mathrm{R} 1)^{*}$ For any polyhedron $P$, open covering $\mathcal{V}$ of $P$ and mapping $f: X \rightarrow$ $P$, there exist an $a \in A$ and a mapping $g: X_{a} \rightarrow P$ satisfying $\left(g p_{a}, f\right) \prec \mathcal{V}$.

$(\mathrm{R} 2)^{*}$ For any polyhedron $P$ and open covering $\mathcal{V}$ of $P$, there exists a $\mathcal{V}^{\prime} \in \operatorname{Cov}(P)$ such that, for any $a \in A$ and mappings $g, g^{\prime}: X_{a} \rightarrow P$ which satisfy $\left(g p_{a}, g^{\prime} p_{a}\right) \prec \mathcal{V}^{\prime}$, there exists an $a^{\prime} \geq a$ such that $\left(g p_{a a^{\prime}}, g^{\prime} p_{a a^{\prime}}\right) \prec \mathcal{V}$.

LEMMA 1. For an approximate mapping $\boldsymbol{p}: X \rightarrow \boldsymbol{X},(\mathrm{R} 1)^{*}$ is equivalent to $(\mathrm{R} 1)$ and $(\mathrm{R} 2)^{*}$ is equivalent to $(\mathrm{R} 2)$.

The proof of this statement for gauged systems, given in [7, Remark 2.6], applies to the present more general situation because it uses only properties (A2) and (AS), which are still valid.

Proof of Theorem 7. First assume that $\boldsymbol{p}$ is a resolution. We must show that $\boldsymbol{q}$ satisfies conditions (R1) and (R2). Let $P$ be a polyhedron, $\mathcal{V} \in \operatorname{Cov}(P)$ and let $f: X \rightarrow P$ be a mapping. Since $\boldsymbol{p}$ satisfies (R1), there exists an $a \in A$ such that, for any $a^{\prime} \geq a$, there is a mapping $g: X_{a^{\prime}} \rightarrow P$ such that $\left(f, g p_{a^{\prime}}\right) \prec \mathcal{V}$. Since $s: B \rightarrow A$ is onto, there is a $b \in B$ such that $s(b)=a$. If $b^{\prime} \in B, b^{\prime} \geq b$, then $a^{\prime}=s\left(b^{\prime}\right) \geq s(b)=a$. Therefore, $\left(f, g q_{b^{\prime}}\right)=$ $\left(f, g p_{s\left(b^{\prime}\right)}\right)=\left(f, g p_{a^{\prime}}\right) \prec \mathcal{V}$, as required by (R1). In order to verify (R2), for $P$ and $\mathcal{V}$, choose $\mathcal{V}^{\prime} \in \operatorname{Cov}(P)$ by (R2) applied to $\boldsymbol{p}$. Consider a $b \in B$ and mappings $g, g^{\prime}: Y_{b} \rightarrow P$ such that $\left(g q_{b}, g^{\prime} q_{b}\right) \prec \mathcal{V}^{\prime}$, i.e. $\left(g p_{s(b)}, g^{\prime} p_{s(b)}\right) \prec \mathcal{V}^{\prime}$. By the choice of $\mathcal{V}^{\prime}$, there exists an $a^{\prime} \in A, a^{\prime} \geq s(b)$, such that

$$
\left(g p_{s(b) a^{\prime \prime}}, g^{\prime} p_{s(b) a^{\prime \prime}}\right) \prec \mathcal{V}, \quad \forall a^{\prime \prime} \in A, a^{\prime \prime} \geq a^{\prime} .
$$

Since $s: B \rightarrow A$ is onto, there exists an element $b^{*} \in B$ such that $s\left(b^{*}\right)=a^{\prime}$. By the directedness of $B$, there exists a $b^{\prime} \in B$ such that $b^{\prime} \geq b, b^{*}$. Now assume that $b^{\prime \prime} \in B$ and $b^{\prime \prime} \geq b^{\prime}$. Then $a^{\prime \prime}=s\left(b^{\prime \prime}\right) \geq s\left(b^{\prime}\right) \geq s\left(b^{*}\right)=a^{\prime}$. Therefore, (1) becomes

$$
\left(g q_{b b^{\prime \prime}}, g^{\prime} q_{b b^{\prime \prime}}\right) \prec \mathcal{V}, \quad \forall b^{\prime \prime} \geq b^{\prime},
$$

which is the desired condition (R2).

We now assume that $\boldsymbol{q}$ is a resolution and must show that $\boldsymbol{p}$ has properties (R1) and (R2). In view of Lemma 1, it suffices to show that $\boldsymbol{p}$ has properties $(\mathrm{R} 1)^{*}$ and $(\mathrm{R} 2)^{*}$. Let $P$ be a polyhedron, $\mathcal{V} \in \operatorname{Cov}(P)$ a covering and $f: X \rightarrow P$ a mapping. By $(\mathrm{R} 1)^{*}$ for $\boldsymbol{q}$, there exist a $b \in B$ and a mapping $g: Y_{b} \rightarrow P$ such that $\left(f, g q_{b}\right) \prec \mathcal{V}$. Putting $a=s(b)$, we have $X_{a}=Y_{b}$, 
$p_{a}=q_{b}$ and therefore, $g: X_{a} \rightarrow P$ has the property required by (R1)* for $\boldsymbol{p}$.

In order to verify condition $(\mathrm{R} 2)^{*}$ for $\boldsymbol{p}$, consider again a polyhedron $P$ and a covering $\mathcal{V} \in \operatorname{Cov}(P)$. Choose $\mathcal{V}^{\prime} \in \operatorname{Cov}(P)$ by applying $(\mathrm{R} 2)^{*}$ to $\boldsymbol{q}$. We claim that $\mathcal{V}^{\prime}$ satisfies $(\mathrm{R} 2)^{*}$ also for $\boldsymbol{p}$. Indeed, let $a \in A$ and let $g, g^{\prime}: X_{a} \rightarrow P$ be mappings such that $\left(g p_{a}, g^{\prime} p_{a}\right) \prec \mathcal{V}^{\prime}$. Choose $b \in B$ so that $s(b)=a$. Then, by the choice of $\mathcal{V}^{\prime}$, there exists a $b^{\prime} \geq b$ such that $\left(g q_{b b^{\prime}}, g^{\prime} q_{b b^{\prime}}\right) \prec \mathcal{V}$. Clearly, $a^{\prime}=s\left(b^{\prime}\right)$ has the property required by $(\mathrm{R} 1)^{*}$ for $\boldsymbol{p}$.

Theorem 8. Let $\boldsymbol{p}: X \rightarrow \boldsymbol{X}$ be an approximate resolution. If all the spaces $X_{a}, a \in A$, are Tikhonov spaces and $X$ is topologically complete (i.e. admits a complete uniform structure), then $\boldsymbol{p}$ is a limit.

Proof. By Theorem 7, the induced approximate mapping $q: X \rightarrow \mathcal{X}$ is a gauged approximate resolution. By Theorem 1(ii), the members of $\mathcal{X}$ are Tikhonov spaces. Therefore, by [7, Theorem 3.1], $\boldsymbol{q}$ is a limit. Now, Theorem 4 yields the desired conclusion that also $\boldsymbol{p}$ is a limit.

Theorem 8 explains the assertion made in the Introduction that approximate resolutions can be viewed as a special case of inverse limits. The converse does not hold even for limits of inverse sequences of metric spaces (see, e.g. [6, I, 6.3, Remark 4] or [7, Example 3.2]).

The next theorem shows that in the compact situation approximate resolutions coincide with limits.

Theorem 9. Let $\boldsymbol{p}: X \rightarrow \boldsymbol{X}$ be an approximate mapping and let all $X_{a}$, $a \in A$, be compact Hausdorff spaces. If $\boldsymbol{p}$ is an approximate resolution and $X$ is topologically complete, e.g. if it is paracompact, then $\boldsymbol{p}$ is a limit and $X$ is compact. Conversely, if $\boldsymbol{p}$ is a limit, then $X$ is a compact Hausdorff space and $\boldsymbol{p}$ is an approximate resolution.

Proof. The first assertion follows from Theorems 8 and 6 . For the second assertion, consider the induced approximate mapping $\boldsymbol{q}: X \rightarrow \mathcal{X}$. By Theorem 4, $\boldsymbol{q}$ is a limit. By [7, Theorem 4.2], $\boldsymbol{q}$ is a gauged approximate resolution. Consequently, Theorem 7 proves that $\boldsymbol{p}$ is an approximate resolution.

Re mark 3. In the analogue of the first assertion for gauged resolutions [7, Theorem 4.21], the assumption that $X$ is topologically complete was erroneously omitted. That such a condition is indeed needed follows from this result of I. Lončar [2, Theorem 3.14]. A normal space $X$ is countably compact if and only if it admits a resolution $\boldsymbol{p}: X \rightarrow \boldsymbol{X}$ all of whose members $X_{a}$ are metric compacta. E.g. the long line $\left\{\alpha \mid \alpha<\omega_{1}\right\}$ is a countably compact normal space and therefore admits a resolution formed 
by metric compacta. On the other hand, it cannot be a limit of compact spaces because it is not compact.

5. Characterizing approximate resolutions. In [7, Theorem 2.8], gauged approximate resolutions were characterized as approximate mappings $\boldsymbol{p}: X \rightarrow \mathcal{X}$ having the following two properties:

(B1) For any $\mathcal{U} \in \operatorname{Cov}(X)$, there exists an $a \in A$ such that for any $a^{\prime} \geq a$ there exists a $\mathcal{W} \in \operatorname{Cov}\left(X_{a^{\prime}}\right)$ for which $\left(p_{a^{\prime}}\right)^{-1}(\mathcal{W}) \prec \mathcal{U}$.

(B2) For any $a \in A$ and $\mathcal{W} \in \operatorname{Cov}\left(X_{a}\right)$, there exists an $a^{\prime} \geq a$ such that $p_{a a^{\prime \prime}}\left(X_{a^{\prime \prime}}\right) \subseteq \operatorname{st}\left(p_{a}(X), \mathcal{W}\right)$, for each $a^{\prime \prime} \geq a^{\prime}$.

Properties (B1) and (B2) are usually easier to verify than properties (R1) and (R2). Therefore, the following theorem is useful.

TheOREM 10. An approximate mapping $\boldsymbol{p}: X \rightarrow \boldsymbol{X}$ is an approximate resolution if and only if it has properties (B1) and (B2).

We precede the proof by introducing formally weaker, but equivalent versions of the above properties.

$(\mathrm{B} 1)^{*}$ For any $\mathcal{U} \in \operatorname{Cov}(X)$, there exist an $a \in A$ and a $\mathcal{W} \in \operatorname{Cov}\left(X_{a}\right)$ such that $p_{a}^{-1}(\mathcal{W}) \prec \mathcal{U}$.

$(\mathrm{B} 2)^{*}$ For any $a \in A$ and $\mathcal{W} \in \operatorname{Cov}\left(X_{a}\right)$, there exists an $a^{\prime} \geq a$ such that $p_{a a^{\prime}}\left(X_{a^{\prime}}\right) \subseteq \operatorname{st}\left(p_{a}(X), \mathcal{W}\right)$.

Notice that the same properties $(\mathrm{B} 1)^{*}$ and $(\mathrm{B} 2)^{*}$ appear in [7]. On the contrary, properties denoted in [7] by (B1) and (B2) involve meshes and therefore differ from the present properties (B1) and (B2).

Lemma 2. For an approximate mapping $\boldsymbol{p}: X \rightarrow \boldsymbol{X},(\mathrm{B} 1)^{*}$ is equivalent to $(\mathrm{B} 1)$ and $(\mathrm{B} 2)^{*}$ is equivalent to $(\mathrm{B} 2)$.

In the proof we need this simple fact.

Lemma 3. Let $f, g: X \rightarrow Y$ be mappings and let $\mathcal{U} \in \operatorname{Cov}(X), \mathcal{V}, \mathcal{W} \in$ $\operatorname{Cov}(Y)$. If $f^{-1}(\mathcal{V}) \prec \mathcal{U}$, $\operatorname{st}(\mathcal{W}) \prec \mathcal{V}$ and $(f, g) \prec \mathcal{W}$, then $g^{-1}(\mathcal{W}) \prec \mathcal{U}$.

Proof of Lemma 2. That (B1) implies (B1)* and (B2) implies (B2)* is obvious. Now assume that $\boldsymbol{p}=\left(p_{a}\right)$ has property $(\mathrm{B} 1)^{*}$. Then for a given $\mathcal{U} \in \operatorname{Cov}(X)$, there exist an $a^{*} \in A$ and a $\mathcal{V} \in \operatorname{Cov}\left(X_{a^{*}}\right)$ such that $p_{a^{*}}^{-1}(\mathcal{V}) \prec$ $\mathcal{U}$. Choose a $\mathcal{W}^{\prime} \in \operatorname{Cov}\left(X_{a^{*}}\right)$ such that $\operatorname{st}\left(\mathcal{W}^{\prime}\right) \prec \mathcal{V}$. By (AS), there exists an $a \geq a^{*}$ such that for any $a^{\prime} \geq a$ one has $\left(p_{a^{*}}, p_{a^{*} a^{\prime}} p_{a^{\prime}}\right) \prec \mathcal{W}^{\prime}$. Then Lemma 3, applied to $p_{a}^{*}, p_{a^{*} a^{\prime}} p_{a^{\prime}}$, yields $\left(p_{a^{*} a^{\prime}} p_{a^{\prime}}\right)^{-1}=\left(p_{a^{\prime}}\right)^{-1}\left(p_{a^{*} a^{\prime}}\right)^{-1}\left(\mathcal{W}^{\prime}\right)$ $\prec \mathcal{U}$. Therefore, if we put $\mathcal{W}=\left(p_{a^{*} a^{\prime}}\right)^{-1}\left(\mathcal{W}^{\prime}\right) \in \operatorname{Cov}\left(X_{a^{\prime}}\right)$, we obtain the desired conclusion $\left(p_{a^{\prime}}\right)^{-1}(\mathcal{W}) \prec \mathcal{U}$. 
To show that (B2)* implies (B2), consider an $a \in A$ and a $\mathcal{V} \in \operatorname{Cov}\left(X_{a}\right)$. Choose a $\mathcal{W}$ so fine that $\operatorname{st}^{2}(\mathcal{W})$ refines $\mathcal{V}$. By $(\mathrm{A} 2)$ and $(\mathrm{AS})$, there exists an $a^{*} \geq a$ such that

$$
\begin{aligned}
\left(p_{a a_{1}} p_{a_{1} a_{2}}, p_{a a_{2}}\right) & \prec \mathcal{W}, & & a^{*} \leq a_{1} \leq a_{2}, \\
\left(p_{a a_{1}} p_{a_{1}}, p_{a}\right) & \prec \mathcal{W}, & & a^{*} \leq a_{1} .
\end{aligned}
$$

Put $\mathcal{W}^{*}=\left(p_{a a^{*}}\right)^{-1}(\mathcal{W})$. Now apply $(\mathrm{B} 2)^{*}$ to $a^{*}$ and $\mathcal{W}^{*}$. One obtains an element $a^{\prime} \geq a^{*}$ such that

$$
p_{a^{*} a^{\prime}}\left(X_{a^{\prime}}\right) \subseteq \operatorname{st}\left(p_{a^{*}}(X), \mathcal{W}^{*}\right)
$$

Note that this inclusion implies

$$
p_{a a^{*}} p_{a^{*} a^{\prime}}\left(X_{a^{\prime}}\right) \subseteq \operatorname{st}\left(p_{a a^{*}} p_{a^{*}}(X), \mathcal{W}\right) .
$$

If $a^{\prime \prime} \geq a^{\prime}$ and $x_{a^{\prime \prime}} \in X_{a^{\prime \prime}},(2)$ yields a set $W_{1} \in \mathcal{W}$ such that

$$
p_{a a^{\prime}} p_{a^{\prime} a^{\prime \prime}}\left(x_{a^{\prime \prime}}\right), p_{a^{\prime \prime}}\left(x_{a^{\prime \prime}}\right) \in W_{1} .
$$

For $x_{a^{\prime}}=p_{a^{\prime} a^{\prime \prime}}\left(x_{a^{\prime \prime}}\right) \in X_{a^{\prime}},(1)$ yields a $W_{2} \in \mathcal{W}$ such that

$$
p_{a a^{*}} p_{a^{*} a^{\prime}}\left(x_{a^{\prime}}\right), p_{a a^{\prime}}\left(x_{a^{\prime}}\right) \in W_{2} .
$$

Since $p_{a^{*} a^{\prime}}\left(x_{a^{\prime}}\right) \in p_{a^{*} a^{\prime}} X_{a^{\prime}},(4)$ yields an $x \in X$ and a $W_{3} \in \mathcal{W}$ such that

$$
p_{a a^{*}} p_{a^{*} a^{\prime}}\left(x_{a^{\prime}}\right), p_{a a^{*}} p_{a^{*}}(x) \in W_{3} .
$$

Finally, (2) yields a $W_{4} \in \mathcal{W}$ such that

$$
p_{a a^{*}} p_{a^{*}}(x), p_{a}(x) \in W_{4} \in \mathcal{W} .
$$

Since $\operatorname{st}^{2}(\mathcal{W})$ refines $\mathcal{V}$, there is a $V \in \mathcal{V}$ such that $W_{1} \cup \ldots \cup W_{4} \subseteq V$. Therefore,

$$
p_{a a^{\prime \prime}}\left(x_{a^{\prime \prime}}\right) \in \operatorname{st}\left(p_{a}(x), \mathcal{V}\right) \subseteq \operatorname{st}\left(p_{a}(X), \mathcal{V}\right)
$$

We have thus proved the desired relation

$$
p_{a a^{\prime \prime}}\left(X_{a^{\prime \prime}}\right) \subseteq \operatorname{st}\left(p_{a}(X), \mathcal{V}\right) .
$$

In the proof of Theorem 10 we also need the following lemma.

Lemma 4. Let $\boldsymbol{p}: X \rightarrow \boldsymbol{X}$ be an approximate mapping and let $\boldsymbol{q}: X \rightarrow \mathcal{X}$ be an induced approximate mapping. Then $\boldsymbol{p}$ has properties (B1), (B2) if and only if $\boldsymbol{q}$ has properties (B1), (B2) in the sense of [7].

Proof. First assume that $\boldsymbol{p}$ has property (B1). Then, for $\mathcal{U} \in \operatorname{Cov}(X)$, there is an $a \in A$ and a $\mathcal{V} \in \operatorname{Cov}\left(X_{a}\right)$ such that $\left(p_{a}\right)^{-1}(\mathcal{V}) \prec \mathcal{U}$. By Theorem 1(iii), there is an element $b \in B$ such that $a=s(b)$. Therefore, $q_{b}=p_{a}$ and we obtain

$$
\left(q_{b}\right)^{-1}(\mathcal{V}) \prec \mathcal{U}
$$

which is $(\mathrm{B} 1)^{*}$ for $\boldsymbol{q}$. By $[7$, Remark 2.9], this implies (B1) for $\boldsymbol{q}$ (in the sense of $[7])$. 
If $\boldsymbol{p}$ has property (B2), then for any $b \in B$ and $\mathcal{U} \in \operatorname{Cov}\left(Y_{b}\right)$, we have $a=s(b) \in A$ and $Y_{b}=X_{a}$. Applying (B2) to $a$ and $\mathcal{U} \in \operatorname{Cov}\left(X_{a}\right)$, we obtain an $a^{\prime} \geq a$ such that

$$
p_{a a^{\prime \prime}}\left(X_{a^{\prime \prime}}\right) \subseteq \operatorname{st}\left(p_{a}(X), \mathcal{U}\right), \quad \forall a^{\prime \prime} \geq a^{\prime} .
$$

Since $s: B \rightarrow A$ is onto, there exists a $b^{*} \in B$ such that $s\left(b^{*}\right)=a^{\prime}$. Now choose for $b^{\prime}$ any element of $B$ such that $b^{\prime} \geq b, b^{*}$. If $b^{\prime \prime} \geq b^{\prime}$, we see that $a^{\prime \prime}=s\left(b^{\prime \prime}\right) \geq a^{\prime}$ and thus $q_{b b^{\prime \prime}}=p_{a a^{\prime \prime}}, q_{b^{\prime \prime}}=p_{a^{\prime \prime}}$. Therefore, (6) becomes

$$
q_{b b^{\prime \prime}}\left(Y_{b^{\prime \prime}}\right) \subseteq \operatorname{st}\left(q_{b}(X), \mathcal{U}\right), \quad \forall b^{\prime \prime} \geq b^{\prime} .
$$

This establishes property (B2) ${ }^{* * *}$ of [7], which is equivalent to (B2) of [7] (see [7, Remark 2.10]).

Now assume that $\boldsymbol{q}$ has properties (B1) and (B2) of [7]. By Lemma 2, it suffices to prove that $\boldsymbol{p}$ has properties (B1)* and (B2)*. If $\mathcal{U} \in \operatorname{Cov}(X)$, $(\mathrm{B} 1)^{*}$ for $\boldsymbol{q}$ (which is equivalent to (B1) of [7] by [7, Remark 2.9]) yields a $b \in B$ and a $\mathcal{V} \in \operatorname{Cov}\left(Y_{b}\right)$ which satisfy (5). However, this yields the analogous relation for $a=s(b)$, which is property (B1)* for $\boldsymbol{p}$. Now let $a \in A$, $\mathcal{V} \in \operatorname{Cov}\left(X_{a}\right)$. Choose $b \in B$ so that $s(b)=a$. Then (B2)* for $\boldsymbol{q}$ (which is equivalent to (B2) of [7] by [7, Remark 2.10]) yields a $b^{\prime} \geq b$ such that $q_{b b^{\prime}}\left(Y_{b^{\prime}}\right) \subseteq \operatorname{st}\left(q_{b}(X), \mathcal{V}\right)$. Putting $a^{\prime}=s\left(b^{\prime}\right)$, one obtains condition $(\mathrm{B} 2)^{*}$ for $\boldsymbol{p}$.

Proof of Theorem 10. Let $\boldsymbol{q}: X \rightarrow \mathcal{X}$ be an induced approximate mapping. If $\boldsymbol{p}$ is an approximate resolution, then $\boldsymbol{q}$ is a gauged approximate resolution (Theorem 4). Therefore, [7, Theorem 2.8] shows that $\boldsymbol{q}$ has properties (B1) and (B2). Now Lemma 4 yields the desired conclusion that also $\boldsymbol{p}$ has these properties. Conversely, if $\boldsymbol{p}$ has properties (B1), (B2), then so does $\boldsymbol{q}$, because of Lemma 4. Therefore, by [7, Theorem 2.8], $\boldsymbol{q}$ is a gauged approximate resolution. Now Theorem 7 yields the desired conclusion that $\boldsymbol{p}$ is an approximate resolution.

Theorem 11. A topological space has covering dimension $\operatorname{dim} X \leq n$ if and only if $X$ admits an approximate resolution $\boldsymbol{p}: X \rightarrow \boldsymbol{X}$ consisting of polyhedra $X_{a}$ of dimension $\operatorname{dim} X_{a} \leq n$.

Proof. That the condition is necessary follows immediately from property (B1). The sufficiency was proved by T. Watanabe [10], who produced a gauged approximate resolution with the desired properties.

In a similar way one can see that recent results on $\wp$-like spaces [4] also hold for approximate resolutions as defined in the present paper. 


\section{References}

[1] M. G. Charalambous, Approximate inverse systems of uniform spaces and an application of inverse systems, Comment. Math. Univ. Carolin. 32 (1991), 551-565.

[2] I. Lončar, Some results on resolution of spaces, Rad Jugosl. Akad. Znan. Umjet. Mat. Znan. 428 (6) (1987), 37-49.

[3] S. Mardešić, Approximate polyhedra, resolutions of maps and shape fibrations, Fund. Math. 114 (1981), 53-78.

[4] S. Mardešić and V. Matijević, $\mathcal{P}$-like spaces are limits of approximate $\mathcal{P}$-resolutions, Topology Appl. 45 (1992), 189-202.

[5] S. Mardešić and L. R. Rubin, Approximate inverse systems of compacta and covering dimension, Pacific J. Math. 138 (1989), 129-144.

[6] S. Mardešić and J. Segal, Shape Theory, North-Holland, Amsterdam 1982.

[7] S. Mardešić and T. Watanabe, Approximate resolutions of spaces and mappings, Glas. Mat. 24 (1989), 583-633.

[8] V. Matijević and N. Uglešić, A new approach to the theory of approximate resolutions, preprint.

[9] T. Watanabe, Approximative shape I, Tsukuba J. Math. 11 (1987), 17-59.

[10] -, Approximate resolutions and covering dimension, Topology Appl. 38 (1991), $147-154$

DEPARTMENT OF MATHEMATICS

UNIVERSITY OF ZAGREB

BIJENIČKA CESTA 30

41000 ZAGREB, CROATIA

Received 5 May 1992;

in revised form 28 October 1992 